\title{
Restoring a Fractured Endodontically Treated Incisor using Fiber Post: A Case Report
}

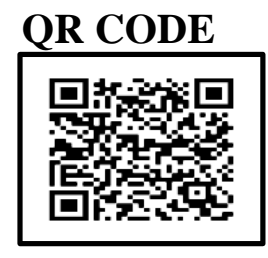

\section{GAYATHRI KM', PRASAD PK*1}

Coronal fractures of the anterior teeth arising after endodontic treatment are sequelae of dental trauma. Endodontically treated teeth presents a higher risk of biomechanical failure than vital teeth. Posts are needed for restoring teeth with insufficient coronal tooth structure to retain a core for definitive restoration. Fiber posts are more easily and safely removed "by hollowing them out from the inside". This present case report depicts a 23 year old male patient with fracture of tooth number 21 which had undergone an RCT 8-10 months ago and was restored using fiber post.

KEYWORDS: Endodontics, Fracture, Post, Restoration

\section{INTRODUCTION}

In comparison to vital teeth, endodontically treated teeth pose in increased risk of biomechanical failure, and hence, posts are needed for restoring such teeth especially those having insufficient coronal tooth structure to retain a core for further restoration. ${ }^{1}$ The selection of an appropriate post material plays a significant impact in the biomechanical performance and an ideal post material should have acceptable modulus of elasticity, compressive strength and thermal expansion. In addition, the esthetics should be similar to those of dentin and it should be fully capable to bond to root dentin and without causing any harm to the dentinal tissue. ${ }^{2}$

However, the only material that can substantiate all these properties can be none other than dentin. Therefore, researchers are constantly finding a post material which has properties almost similar to dentin and hence, a most of the fiber posts contain either carbon fiber or quartz fiber and they have the ability to flex with the root when under stress.

In routine clinical practice, the usage of fiber posts are finding an increased application. Studies document that fiber posts strengthen the root when used with a resin luting cement with several short-term clinical studies report a high success rate of these posts. ${ }^{3}$ However, an important caveat regarding fiber posts is whether they should allow movement of the core during function or parafunction as if a post (which is thinner in diameter) has the same modulus of elasticity as the dentin, shall flex more under a load and lead to leakage under the crown and buildup area.
Fiber posts (excepting a South American post design that has a metal wire running through its long axis) are more easily and safely removed "by hollowing them out from the inside" and hence do not require much time and effort. 4,5

Another clinical drawback of a fiber post is its radiolucency, and hence, is impossible to detect radiographically and black color of the x-ray film and can go undetected at times.

This present case report depicts a 23 year old male patient with fracture of tooth number 21 which had undergone an RCT 8-10 months ago and was restored using fiber post.

\section{CASE REPORT}

A 23 year-old male patient reported to the our satellite clinic due to accidental trauma of the left central incisor. On examination, it was found that tooth 21 had undergone root canal treatment 8-10 months ago. However, tooth 21 was asymptomatic and the clinical crown was $<2 \mathrm{~mm}$. (Figure. 1)

The radiographic examination of tooth 21 revealed conical root canal with well condensed gutta percha filling extending $0.5 \mathrm{~mm}$ short of the radiographic apex. No periapical changes were noted in relation to tooth 21 .

An occlusal model evaluation was done to assess the amount of space available for the post endodontic restoration to restore the tooth to function. 

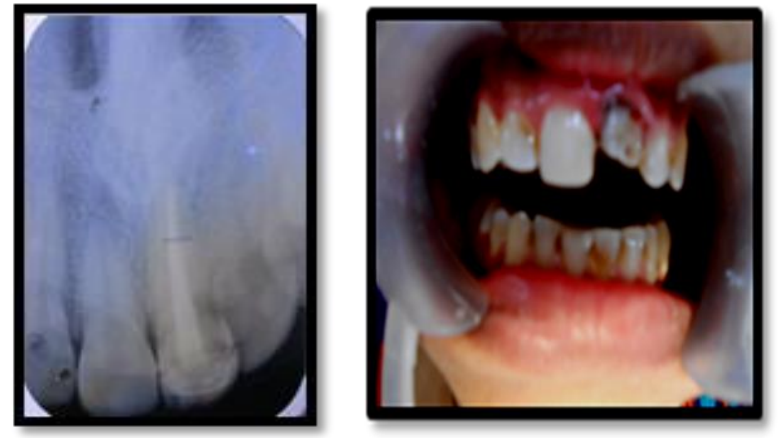

Figure 1. Pre-operative Photograph

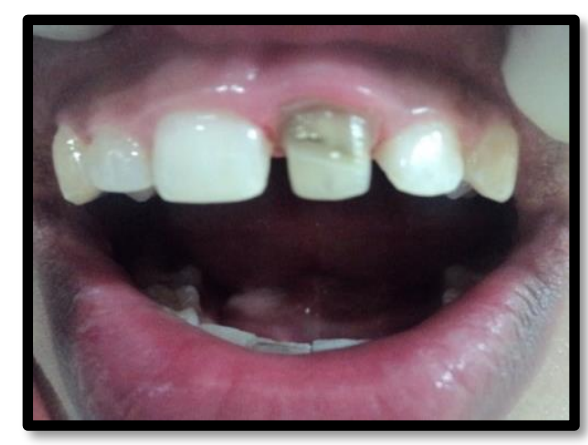

Figure 3. Core Build up and Crown Preparation

In this procedure, tooth was isolated and gutta percha was removed with a small starter drill and the canal is prepared with the matching sized post drills and posts. All remnants of gutta percha must be removed from the walls of the post space to facilitate bonding. The fiber post was then inserted with the paracore material (Coltene) and shortened to the height of the core with a diamond bur before the bonding procedure is started.(Figure 2)

Core build and crown lengthening process was done by removing $2 \mathrm{~mm}$ of keratinized gingival and underlying bone(Figure 3). Then the tissue was left for 1-2 week for healing by secondary healing process and maintaining the appropriate biological width.

Impressions were made, upper with the putty and lower with the alginate impression material. Casts were poured, and crowns were fabricated, after shade matching with $2 \mathrm{M} 2$ shade [VITA]. And then crowns were luted by GIC [luting type](Figure 4).

\section{DISCUSSION}

A trauma which leads to the fracture of anterior tooth/teeth is indeed a tragic experience for a patient

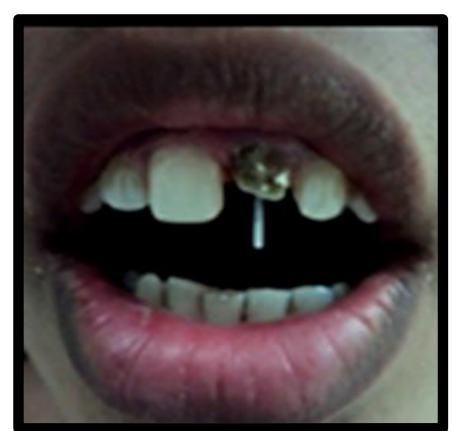

Figure 2. Insertion of Fiber Post

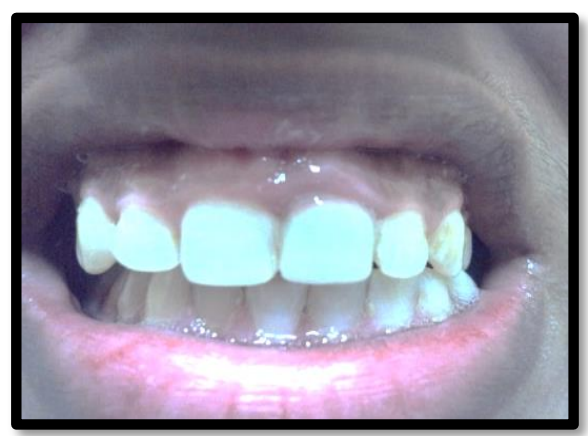

Figure 4. Post-operative photograph

which requires immediate attention. This trauma also has the ability to cause a psychological effect of the trauma to the patient due to pain and poor aesthetics. Studies have documented a positive emotional and social response from the patient to the preservation of natural tooth structure. ${ }^{6}$ A considerable challenge is to restore the functional, esthetic, and biologic restoration of the fractured. 7 There are many different treatment modalities for restoration of traumatized teeth such as composite resin restorations with or without pins and prosthetic repair. ${ }^{8}$

The number of endodontic procedures across the globe has increased in the past decade with highly predictable(positive) results and hence, restoration of teeth after endodontic treatment is becoming an integral part of restorative practice in dentistry. When a considerable amount of tooth structure has been lost as in case trauma or else, special techniques are needed to restore such a tooth. The teeth with minimal vertical tooth structure remaining for crown margins are subjected to flexion forces under function. As less cervical tooth structure is available, cervical stiffening from a more stiff post is needed to protect the crown margins and resist leakage. ${ }^{9}$ At the same 
time, force dissipation from a more resilient post is needed to resist root fracture. The use of fiber post with core is also recommended as it can create a monobloc which is a multi layered structure with no weak interlayer interfaces. ${ }^{10}$

\section{REFERENCES}

1. Gogna R, Jagadish S, Shashikala K, Keshava BS. Restoration of badly broken, endodontically treated posterior teeth J Conserv Dent. 2009; 12(3): 123-8.

2. Cheung W. A review of the management of endodontically treated teeth. Post, core and the final restoration. J Am Dent Assoc. 2005;136(5):611-9.

3. Bartlett SO. Construction of detached core crowns for pulpless teeth in only two sittings. J Am Dent Assoc. 1968;77:843-5.

4. Cheung W. Properties of and important concepts in restoring the endodontically treated teeth. Dent Asia.2004:40-7.
5. Cheung W. A review of the management of Endodontically treated teeth Post, core and the final restoration. J Am Dent Assoc. 2005;136:611-9.

6. Hegde RJ. Tooth fragment reattachment-an esthetic alternative: Report of a case. Journal of Indian Society of Pedodontics and Preventive Dentistry (2003);21(3): 117-9.

7. Murchison DF. Incisal edge reattachment: Indications for use and clinical technique. British Dental Journal 99;186:614-9.

8. Ozel E. Two-year follow-up of fractured anterior teeth restored with direct composite resin: Report of three cases. Dental Traumatology 2008:24(5): 589-92. 9. Torabi K, Fattahi F. Fracture resistance of endodontically treated teeth restored by different FRC posts: an in vitro study. Indian Journal of Dental Research 2009,20(3):282-7.

10. Hayashi M. Fracture resistance of pulpless teeth restored with post-cores and crowns. Dental Materials 2006;22(5):477- 85 .
Source of support: Nil, Conflict of interest: None declared
Cite this article as:

Gayathri KM, Prasad PK. Restoring a Fractured Endodontically Treated Incisor

Using Fiber Post: A Case Report. Int Healthc Res J. 2020;3(11):355-357. https://doi.org/10.26440/IHRJ/0311.02321 